This is an electronic reprint of the original article. This reprint may differ from the original in pagination and typographic detail.

\author{
Author(s): Doncel, M.; Cederwall, B.; Qi, C.; Li, H.; Jakobsson, Ulrika; Auranen, Kalle; Bönig, S.; \\ Drummond, M.; Grahn, Tuomas; Greenlees, Paul; Herzan, Andrej; Joss, D. T.; Julin, \\ Rauno; Juutinen, Sakari; Konki, Joonas; Kröll, T.; Leino, Matti; McPeake, C.; O'Donnell, \\ D.; Page, R. D.; Pakarinen, Janne; Partanen, Jari; Peura, Pauli; Rahkila, Panu; \\ Ruotsalainen, Panu; Sandzelius, Mikael; Sarén, Jan; Sayğı, B.; Scholey, Catherine; Sorri, \\ Title: Lifetime measurements of excited states in $162 \mathrm{~W}$ and $164 \mathrm{~W}$ and the evolution of \\ collectivity in rare-earth nuclei
}

Year: $\quad 2017$

Version:

Please cite the original version:

Doncel, M., Cederwall, B., Qi, C., Li, H., Jakobsson, U., Auranen, K., Bönig, S., Drummond, M., Grahn, T., Greenlees, P., Herzan, A., Joss, D. T., Julin, R., Juutinen, S., Konki, J., Kröll, T., Leino, M., McPeake, C., O'Donnell, D., . . Uusitalo, J. (2017). Lifetime measurements of excited states in $162 \mathrm{~W}$ and $164 \mathrm{~W}$ and the evolution of collectivity in rare-earth nuclei. Physical Review C, 95(4), Article 044321. https://doi.org/10.1103/PhysRevC.95.044321

All material supplied via JYX is protected by copyright and other intellectual property rights, and duplication or sale of all or part of any of the repository collections is not permitted, except that material may be duplicated by you for your research use or educational purposes in electronic or print form. You must obtain permission for any other use. Electronic or print copies may not be offered, whether for sale or otherwise to anyone who is not an authorised user. 


\title{
Lifetime measurements of excited states in ${ }^{162} \mathrm{~W}$ and ${ }^{164} \mathrm{~W}$ and the evolution of collectivity in rare-earth nuclei
}

\author{
M. Doncel, ${ }^{1,2}$ B. Cederwall, ${ }^{1}$ C. Qii, ${ }^{1}$ H. Li ${ }^{1,3}$ U. Jakobsson, ${ }^{1,4}$ K. Auranen, ${ }^{5,6}$ S. Bönig, ${ }^{7}$ M. C. Drummond, ${ }^{2}$ T. Grahn, ${ }^{5}$ \\ P. T. Greenlees, ${ }^{5}$ A. Herzan, ${ }^{2,5}$ D. T. Joss, ${ }^{2}$ R. Julin, ${ }^{5}$ S. Juutinen, ${ }^{5}$ J. Konki, ${ }^{5}$ T. Kröll, ${ }^{7}$ M. Leino, ${ }^{5}$ C. McPeake, ${ }^{2}$ \\ D. O’Donnell, ${ }^{2}$ R. D. Page, ${ }^{2}$ J. Pakarinen, ${ }^{5}$ J. Partanen, ${ }^{5}$ P. Peura, ${ }^{5,8}$ P. Rahkila, ${ }^{5}$ P. Ruotsalainen, ${ }^{5}$ M. Sandzelius, ${ }^{5}$ J. Sarén, ${ }^{5}$ \\ B. Sayğ ${ }_{1},{ }^{2,9}$ C. Scholey, ${ }^{5}$ J. Sorri, ${ }^{5}$ S. Stolze, ${ }^{5}$ M. J. Taylor, ${ }^{10}$ A. Thornthwaite, ${ }^{2}$ and J. Uusitalo ${ }^{5}$ \\ ${ }^{1}$ Royal Institute of Technology (KTH), 10691 Stockholm, Sweden \\ ${ }^{2}$ Department of Physics, Oliver Lodge Laboratory, University of Liverpool, Liverpool L69 7ZE, United Kingdom \\ ${ }^{3}$ Grand Accélérateur National d'Ions Lourds (GANIL), CEA/DSM-CNRS/IN2P3, F-14076 Caen Cedex 5, France \\ ${ }^{4}$ Laboratory of Radiochemistry, Department of Chemistry, P.O. Box 55, FI-00014 Univeristy of Helsinki, Finland \\ ${ }^{5}$ University of Jyvaskyla, Department of Physics, P.O. Box 35, FI-40014 University of Jyvaskyla, Finland \\ ${ }^{6}$ Physics Division, Argonne National Laboratory, Argonne, Illinois 60439, USA \\ ${ }^{7}$ Institut fur Kernphysik, TU Darmstadt, D-64289 Darmstadt, Germany \\ ${ }^{8}$ Helsinki Institute of Physics, FI-00014 Helsinki, Finland \\ ${ }^{9}$ Ege University, Department of Physics, 35100 Izmir, Turkey \\ ${ }^{10}$ School of Physics and Astronomy, The University of Manchester, Oxford Road, Manchester M13 9PL, United Kingdom
}

(Received 10 February 2017; published 24 April 2017)

\begin{abstract}
Lifetimes of the first excited $2^{+}$states in the extremely neutron-deficient ${ }^{162} \mathrm{~W}$ and ${ }^{164} \mathrm{~W}$ nuclei have been measured using the recoil distance Doppler shift technique. Experimental $B(E 2)$ data for the isotopic chains of hafnium, tungsten, and osmium, from the midshell region near the $\beta$-stability line towards the $N=82$ closed shell and the most neutron-deficient nuclides, are compared with predictions of nuclear deformations and $2_{1}{ }^{+} \rightarrow 0_{\text {g.s. }}{ }^{+}$reduced transition strengths from different classes of state-of-the-art theoretical model calculations. The results reveal striking differences and deficiencies in the predictive power of current nuclear structure models.
\end{abstract}

DOI: 10.1103/PhysRevC.95.044321

\section{INTRODUCTION}

Two properties of the first excited $2^{+}$state in atomic nuclei with even numbers of neutrons and protons, the excitation energy and lifetime (a measure of the electric quadrupole transition strength to the ground state), are fundamental observables in nuclear structure physics. These quantities are often used as a measure of the nuclear ground-state deformation and the degree of collective strength at low excitation energies. Both are important benchmarks for nuclear models. In particular the reduced electric quadrupole transition probability, $B(E 2)$, directly probes the wave functions of the lowest-lying excited states and the ground state.

In general, several nucleons or more away from the magic neutron and proton numbers (which reflect major gaps in the energy level spectrum), an emergence of collectivity is observed. This signals that the wave function spreads to multiple coherent particle-hole components that open, e.g., vibrational or rotational degrees of freedom and is normally associated with a lowering of the first excited $2_{1}{ }^{+}$state energy accompanied by an increase in the $21^{+} \rightarrow 0_{\text {g.s. }}{ }^{+}$ reduced transition strength. A gradual evolution of such $B\left(E 2: 2^{+} \rightarrow 0^{+}\right)$values is then naively expected along an isotopic chain: from spherical systems near closed shells where the $B(E 2)$ strength is at a minimum and governed by the individual single-particle degrees of freedom, via quadrupole surface vibrations around spherical symmetry, to the gradual development of deformation towards well-developed axially symmetric shapes with their associated rotational excitations and maximal $B(E 2)$ values when the Fermi level is in the middle between major shell gaps. Raman et al. [1,2] and earlier Grodzins [3] investigated the global distributions of experimental $B\left(E 2: 2^{+} \rightarrow 0^{+}\right)$values and proposed well-known empirical formulas relating the lifetimes of the first excited $2^{+}$states in even-even nuclei to their excitation energies. Such global fits describe reasonably well the general trends across the Segré chart albeit with large individual variations, presumably due to detailed nuclear structure effects, which must be considered using microscopic models. Raman et al. also investigated the predictive power of a selection of nuclear structure models available around the year 2000 [2].

It has since then been noticed that the $B(E 2)$ values in the rare-earth nuclei around the neutron midshell exhibit a saturating behavior as a function of the neutron number, and it was discussed using a phenomenological approach within the interacting boson approximation (IBA) framework [4]. This saturation effect has been confirmed by recent measurements on the $B(E 2)$ values of neutron-deficient $\mathrm{Hf}$ and $\mathrm{W}$ isotopes [5].

We here present new lifetime measurements that extend the $B\left(E 2: 2^{+} \rightarrow 0^{+}\right)$systematics in the $\mathrm{W}$ isotopic chain to the extremely neutron-deficient isotopes ${ }^{162} \mathrm{~W}$ and ${ }^{164} \mathrm{~W}$ and compare the available data for the neutron-deficient region $(88 \leqslant N \leqslant 104)$ of the Hf, W, and Os isotopic chains with the predictions from current state-of-the art nuclear structure theories. 


\section{EXPERIMENTAL DETAILS}

Excited states in highly neutron-deficient species of primarily the tantalum, tungsten, rhenium, and osmium isotopic chains were populated in fusion-evaporation reactions induced by a ${ }^{78} \mathrm{Kr}$ beam at $380 \mathrm{MeV}$ bombarding energy impinging on a thin, self-supporting, isotopically enriched ${ }^{92}$ Mo metallic target foil. The beam was produced by the K-130 cyclotron at the University of Jyväskylä (JYFL), Finland. The ${ }^{162} \mathrm{~W}$ and ${ }^{164} \mathrm{~W}$ nuclides studied in this work were produced in the $\left({ }^{78} \mathrm{Kr}, 2 \alpha\right)$ and $\left({ }^{78} \mathrm{Kr}, \alpha 2 p\right)$ reaction channels, respectively. The experimental setup consisted of the Jurogam II high-purity germanium detector array [6,7] coupled to the RITU gas-filled recoil separator [8] and the differential plunger for unbound nuclear states (DPUNS) [9]. The DPUNS device was equipped with an isotopically enriched ${ }^{92}$ Mo target foil of areal density $0.6 \mathrm{mg} / \mathrm{cm}^{2}$ and a $1 \mathrm{mg} / \mathrm{cm}^{2}$ thick $\mathrm{Mg}$ degrader foil, which decreased the average velocity of the recoiling fusion residues by approximately $20 \%$. The RITU separator was used to discriminate the beam particles from recoils by measuring both the energy deposited in the multiwire proportional counter (MWPC) and the time-of-flight of recoils measured between the MWPC gas detector and the two double-sided silicon strip detectors (DSSSDs) of the focal plane detector system GREAT [10], in which the fusion residues were implanted. The prompt $\gamma$ rays emitted in delayed coincidence with the recoiling fusion products were measured with Jurogam II, which consisted of 15 phase-I type and 24 segmented clover detectors from the former Euroball detector array [11] arranged in four rings, resulting in a photopeak efficiency of $\sim 6 \%$ at $1.3 \mathrm{MeV} \gamma$-ray energy. Nine different target-to-degrader distances, ranging from 5-8000 $\mu \mathrm{m}$, were used for the lifetime analysis.

\section{METHOD AND ANALYSIS}

The lifetime analysis was performed following the principles of the recoil distance Doppler shift (RDDS) technique [12-14] and the differential decay curve method [15]. The data were analyzed for each target-to-degrader distance using the GRAIN software package [16]. Two different approaches have been considered depending on the statistics that could be obtained for the analysis. In the case of ${ }^{164} \mathrm{~W}$ a $\gamma-\gamma$ coincidence analysis could be performed by considering recoil-gated asymmetric $E_{\gamma}-E_{\gamma}$ matrices in which all detectors were considered for the energy cuts (on the $X$ axis) while only the ten tapered coaxial detectors from the ring located at $133.6^{\circ}$ with respect to the beam direction were considered when producing the spectra of interest (cuts projected onto the $Y$ axis). The lifetime of the first excited $2^{+}$state has been extracted using the following equation:

$$
\tau_{i}(x)=\frac{\left\{C_{0}^{\infty}, A_{t}^{\infty}\right\}-\frac{\left\{C_{0}^{\infty}, A_{0}^{\infty}\right\}}{\left\{C_{0}^{\infty}, B_{0}^{\infty}\right\}}\left\{C_{0}^{\infty}, B_{t}^{\infty}\right\}}{\frac{d}{d x}\left\{C_{0}^{\infty}, A_{0}^{t}\right\}} \frac{1}{v},
$$

where we are considering a $\gamma$-ray cascade from $l_{4} \stackrel{C_{\gamma}}{\longrightarrow}$ $l_{3} \stackrel{B_{\gamma}}{\rightarrow} l_{2} \stackrel{A_{\gamma}}{\rightarrow} l_{1}$ and $l_{i}$ represents the different levels being $l_{2}$ the level of interest in this particular case, $C_{\gamma}$ stands for the gating transition while $B_{\gamma}$ and $A_{\gamma}$ stand for the feeding and depopulating transitions, respectively. $\left\{C_{0}^{\infty}, B_{t}^{\infty}\right\}$ and $\left\{C_{0}^{\infty}, A_{t}^{\infty}\right\}$ are the intensities of $\gamma$-rays emitted after the degrader from the $B_{\gamma}$ and $A_{\gamma}$ transitions, respectively, where a gate on both components of the $C$ transition (before and after the degrader foil) is performed while $\left\{C_{0}^{\infty}, A_{0}^{t}\right\}$ corresponds to the intensity of $\gamma$ rays emitted before the degrader from the $A_{\gamma}$ transition when the same gate is applied. The ratio $\frac{\left\{C_{0}^{\infty}, A_{0}^{\infty}\right\}}{\left\{C_{0}^{\infty}, B_{0}^{\infty}\right\}}$ is taken between the intensity of the depopulating and feeding transitions in the gated spectra, respectively, while $\frac{d}{d x}\left\{C_{0}^{\infty}, A_{0}^{t}\right\}$ is the slope of the intensity as a function of distance (deduced using the code APATHIE [17]) of the $\gamma$ rays emitted before the degrader from the $A_{\gamma}$ transition in the same conditions. The recoil velocity directly after the target was determined to be $v=0.0441(5) c$. The intensities of $\gamma$-ray transitions and $\gamma-\gamma$ coincidences were deduced using the RADWARE data analysis package [18] taking into account detector efficiency and the internal conversion process, here taken from the BrIcc database [19].

For the nucleus ${ }^{162} \mathrm{~W}$ the low production cross section and the resulting low statistics did not allow use of the $\gamma-\gamma$ coincidence technique. However, due to the relatively short half life of ${ }^{162} \mathrm{~W}, 990(30) \mathrm{ms}$ [20] and its large $\alpha$-decay branching ratio of approximately $45 \%$ [21], the analysis could be performed using singles spectra of $\gamma$-ray energies in delayed coincidence with characteristic $\alpha$-decay energies detected in the DSSSD detectors at the focal plane of the RITU mass separator, i.e., by applying the recoil-decay tagging (RDT) method [22-24]. In this way, clean selection of the $\gamma$ rays belonging to ${ }^{162} \mathrm{~W}$ could be obtained [20]. However, when using one-dimensional $\gamma$-ray spectra the excited-state lifetime measurement relies on the capability to determine the detailed feeding pattern. In this particular case, as was pointed out in Refs. [20,25], there is no observed side feeding to the first excited $2^{+}$state in ${ }^{162} \mathrm{~W}$. Therefore, it was sufficient to consider only the feeding $4^{+} \rightarrow 2^{+}$and depopulating $2^{+} \rightarrow 0^{+}$transitions for its lifetime determination, see, e.g., Ref. [26]. The lifetime value obtained for three target-to-degrader distances in the region of sensitivity remained constant within statistical uncertainties, confirming the validity of the method employed. Typical spectra and normalized decay intensities used in the lifetime determination for the first excited $2^{+}$state in ${ }^{162} \mathrm{~W}$ are shown in Figs. 1(a) and 1(b), respectively. Lifetime values of $\tau=27 \pm 11$ ps and $\tau=26 \pm 17$ ps have been deduced for the $2_{1}{ }^{+} \rightarrow 0_{\text {g.s. }}{ }^{+}$transitions in ${ }^{162} \mathrm{~W}$ and ${ }^{164} \mathrm{~W}$, respectively. The results are summarized in Table I.

TABLE I. Energy of the $2^{+} \rightarrow 0^{+}$transitions $\left(E_{\gamma}\right)$, lifetime value $(\tau)$ for the $2_{1}{ }^{+}$state and experimental value deduced for the reduced transition probability $[B(E 2 \downarrow)$ exp $]$ for ${ }^{162} \mathrm{~W}$ and ${ }^{164} \mathrm{~W}$ measured in the present work.

\begin{tabular}{lccc}
\hline \hline Nucleus & $E_{\gamma}(\mathrm{keV})$ & $\tau(\mathrm{ps})$ & $B(E 2 \downarrow)_{\exp }($ W.u. $)$ \\
\hline${ }^{162} \mathrm{~W}$ & 449 & $27(11)$ & $31(13)$ \\
${ }^{164} \mathrm{~W}$ & 332 & $26(17)$ & $150(100)$ \\
\hline \hline
\end{tabular}



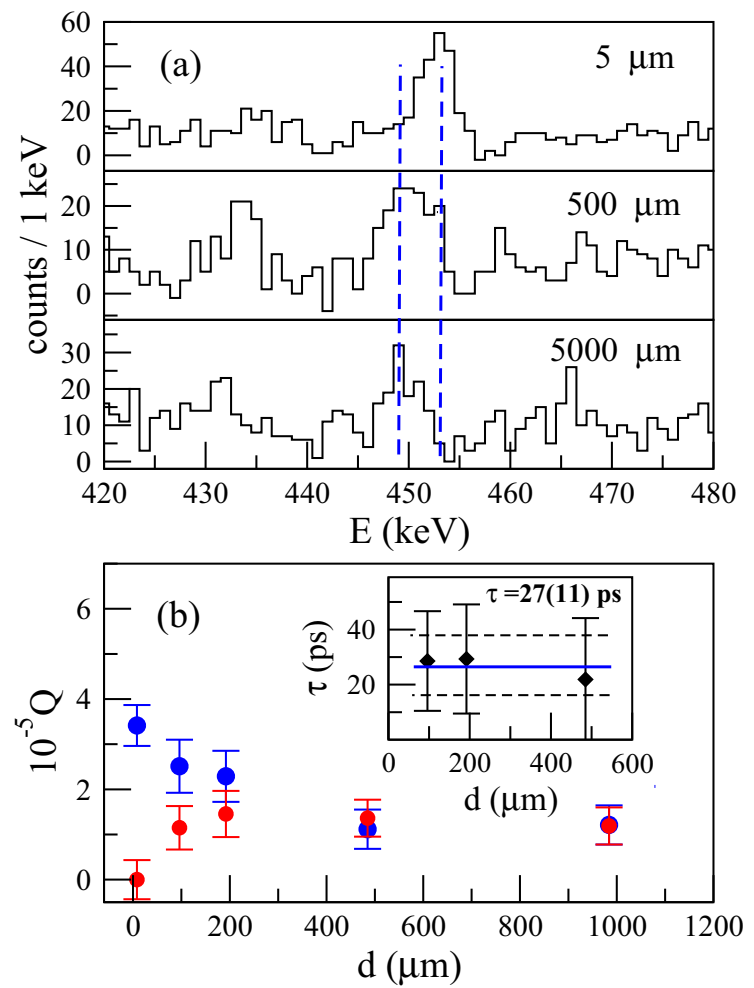

FIG. 1. (a) $\alpha$-tagged background-subtracted spectra used for the lifetime determination in ${ }^{162} \mathrm{~W}$ for three different target-to-degrader distances. (b) Normalized intensity as a function of target-to-degrader distance of $\gamma$ rays from the $2_{1}{ }^{+} \rightarrow 0_{g s}{ }^{+}$transition in ${ }^{162} \mathrm{~W}$ emitted before (red symbols) and after (blue symbols) the degrader foil. Inset: lifetime of the $2^{+}$state evaluated for three target-to-degrader distances in the region-of-sensitivity along with the weighted mean of $27 \mathrm{ps}$ (solid blue line) and its uncertainty \pm 11 ps (dashed black lines).

\section{DISCUSSION}

A region of well-deformed quantum rotors is found in mass $A \approx 170$ rare-earth nuclei, where typical $\beta_{2}$-deformation values are about $0.2-0.3$ (corresponding to ellipsoidal shapes with major/minor axis ratios of approximately $4: 3$ ). This translates into relatively large $B(E 2)$ values, up to several hundred Weisskopf units, due to the simple geometrical relationship between $\beta_{2}$ deformation and $B(E 2)$ strength [27]. In order to illustrate the evolution of collectivity in the $\mathrm{W}$ isotopes, $2_{1}{ }^{+}$excitation energies and $B\left(E 2: 2^{+} \rightarrow 0^{+}\right)$values for neutron numbers ranging from $N=88$ to $N=104$ are shown in Fig. 2 (top and bottom panels, respectively). The experimental $2{ }^{+}$state excitation energies exhibit a smooth decrease as the neutron number approaches the neutron midshell at $N=104$. The experimental reduced transition probabilities follow qualitatively the expected opposite decreasing trend when approaching the $N=82$ closed shell from the neutron midshell at $N=104$, but exhibit a more complex variation as a function of neutron number than that of $E\left(2_{1}{ }^{+}\right)$, reflecting their higher sensitivity to details in the wave function.

In order to investigate the structure of the lightest tungsten isotopes as reflected by our lifetime data on ${ }^{162} \mathrm{~W}$ and ${ }^{164} \mathrm{~W}$ and the evolution of quadrupole collectivity towards stability, we have performed systematic calculations of their ground-state deformations within the macroscopic-microscopic (mac-mic) framework using empirical mean-field potentials as well as within the self-consistent energy density functional mean-field framework. The mac-mic calculations are done using the potential energy surface (PES) approach with the universal Woods-Saxon single-particle potential [28] and two other parameter sets from Refs. [29,30] (Woods-Saxon). See also the global systematic study of Woods-Saxon calculations performed in Ref. [31]. We note that cranked PES calculations for several $\mathrm{W}$ isotopes have been performed previously and used to assign the band configurations [20,25]. We have compared our results with those obtained from the finite-range droplet model (FRDM) calculations reported in Ref [32]. We have also performed Skyrme Hartree-Fock-Bogoliubov calculations with the Sly4 [33] and UNEDF0-2 [34-36] parameters in the transformed harmonic oscillator basis using the code HFBTHO [37]. In addition, we have carried out relativistic mean-field calculations using the code developed by Niksic et al. [38] with the DD-ME2 parameter set [39]. Furthermore, we have compared our results with the results of four large systematic theoretical studies available in the literature based on the HFB24 mass model [40], the deformed quasiparticle random-phase approximation (dQRPA) [41], the coherent state model (CSM) [42], and Hartree-Fock-Bogoliubov beyondmean-field calculations using the generator coordinate method based on the Gogny D1S interaction (HFBD1S-BMF) [43]. The theoretically predicted energies, $E\left(2^{+}\right)$, from the CSM and HFBD1S-BMF calculations are also shown in the top panel of Fig. 2. The dQRPA, CSM, and HFBD1S-BMF compilations include direct calculations of E2 transition strengths for the first excited $2^{+}$states in a large number of even-even nuclei. The dQRPA calculation was performed using the experimental $E\left(2_{1}{ }^{+}\right)$energy as input and effective charges $e_{\pi}=(1+\chi), e_{v}=\chi$, where the effective polarization parameter, $\chi$, is taken to be $\chi=0.25$ [44] in this work while $\chi=0.20$ was used in Ref. [41].

Note that when the relevant quantity available from the theoretical models is the quadrupole deformation parameter $\left(\beta_{2}\right)$ and not the $B(E 2)$ value, a direct comparison with the experimental data can not be performed. With the exception of the dQRPA, CSM, and HFBD1S-BMF calculations, which include direct predictions of $B\left(E 2: 2^{+} \rightarrow 0^{+}\right)$values, we have therefore estimated the theoretical $B(E 2)$ values from the calculated quadrupole deformation in the usual way within the framework of the collective model [2,27]. The experimental $B\left(E 2: 2^{+} \rightarrow 0^{+}\right)$values are compared with values derived from the theoretical calculations in Fig. 2 (bottom panel). $B(E 2)$ values obtained using the Raman-Grodzins empirical formula (Raman) [2] are also included for comparison.

The experimental uncertainty in the $B(E 2)$ value for ${ }^{164} \mathrm{~W}$ is too large to enable a stringent test of the theoretical predictions. For ${ }^{162} \mathrm{~W}$, only the Raman empirical fit is close to the experimental value. In general, strikingly large deviations between the calculations and the experimental data are seen. The theoretical calculations mostly overshoot the experimental values and tend to show the same bell type of variation as a function of neutron number $(N)$, with a maximum at the neutron midshell (as expected). An exception is the 


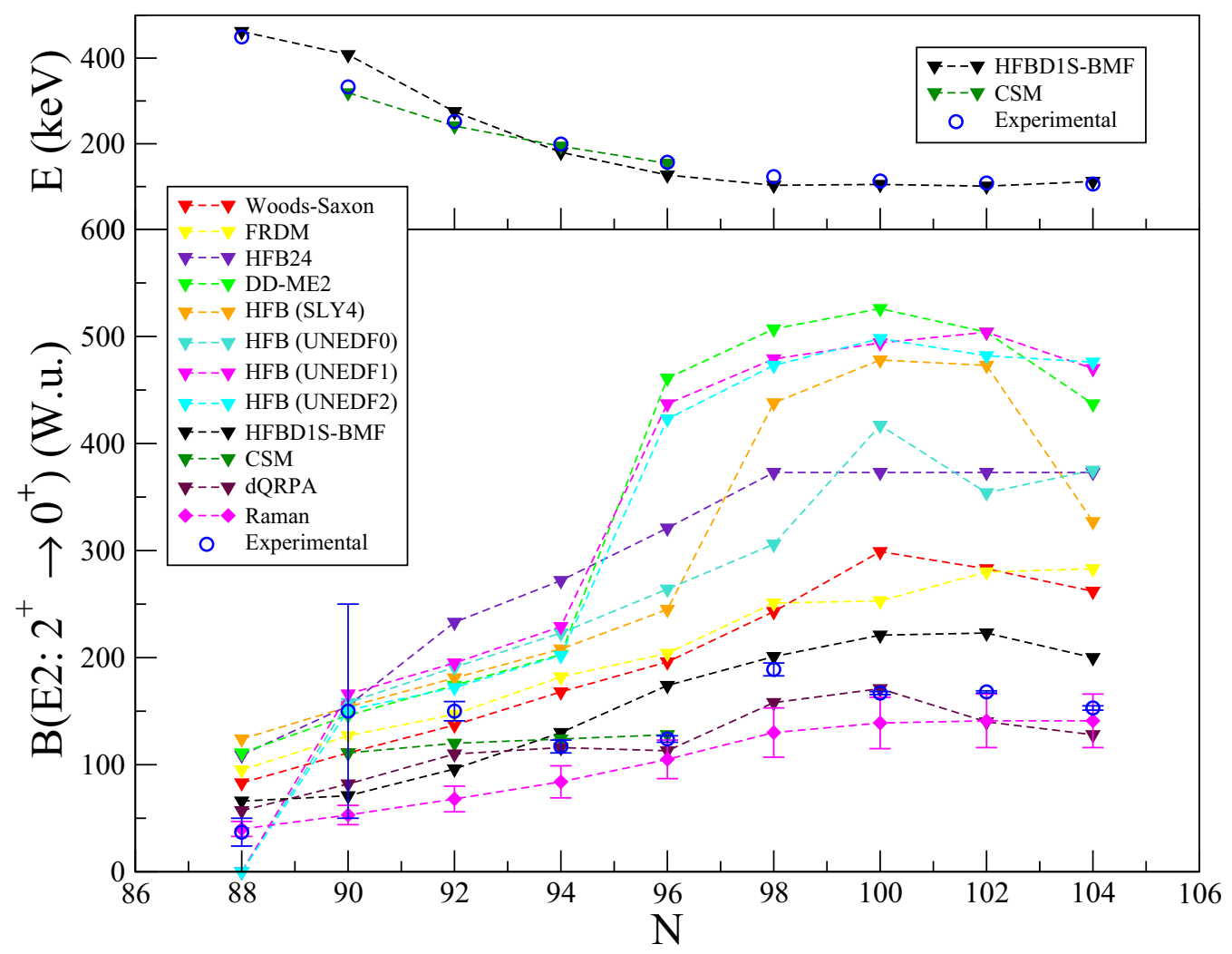

FIG. 2. Top: Evolution of $2_{1}{ }^{+}$energies for $\mathrm{W}$ isotopes as a function of neutron number, $N$. Experimental data are compared with the HFBD1S calculations with beyond-mean-field (BMF) corrections from Ref. [43] and phenomenological CSM calculations from Ref. [42]. Bottom: Reduced transition probabilities (in W.u.) for W isotopes (bottom). The experimental $B(E 2)$ values for ${ }^{162} \mathrm{~W}$ and ${ }^{164} \mathrm{~W}$ have been measured in the present work, ${ }^{166} \mathrm{~W}$ has been taken from Ref. [45] and the rest of the data comes from Ref. [5] and references therein. The results of different theoretical model calculations (see text for details) are shown as symbols connected by dashed lines. $B(E 2)$ values obtained using the Raman-Grodzins empirical formula (Raman) [2] are also shown for comparison.

phenomenological CSM calculations, which produce a rather flat dependence of $B(E 2)$ as a function of $N$. None of the model calculations seems able to reproduce the more subtle variations in the experimental values. The best overall agreement is produced by the CSM, dQRPA, and HFBD1SBMF calculations, followed by the Woods-Saxon and FRDM mac-mic potential models. Note, however, that while the phenomenological CSM model and the dQRPA model use effective charges, which are adjusted to match the experimental data, the HFBD1S-BMF calculation takes a self-consistent approach. The Raman empirical parametrization produces, as expected, a fair agreement on average but also fails to reproduce the variations in the experimental data as a function of neutron number.

The structure of the neutron-deficient isotopes considered is mostly driven by the interplay between protons located in the $h_{11 / 2}$ orbital and neutrons in $h_{9 / 2}$ and $f_{7 / 2}$ orbitals. In Ref. [4] the saturating behavior of the $B(E 2)$ values around midshell is discussed in terms of reduced neutron-proton correlations. Comparing the occupation of single-particle levels and wave functions derived from the nuclear structure models studied in this work it is noticed that the calculated ground-state deformation of those nuclei can also be sensitive to the proton $h_{9 / 2}$ intruder states and the neutron $i_{13 / 2}$ orbitals. A larger deformation is expected from the calculations when those orbitals come down in energy. Hence, the large variations in the different model predictions may be related to differences in the occupation of certain single-particle levels.

In order to investigate whether the large theoretical uncertainties in the predictions of $B\left(E 2 ; 2^{+} \rightarrow 0^{+}\right)$values are a peculiarity of the $\mathrm{W}$ isotopic chain we extended the study to include also the surrounding $\mathrm{Hf}$ and Os isotopic chains. The results are shown in Fig. 3, indicating that the deficiencies found in the predictive power of the theoretical models investigated in this work is not isolated to the $\mathrm{W}$ isotopic chain, but is a more general feature.

The results based on the Skyrme density functional $[33,36,40]$ and relativistic mean-field [38] theory show especially poor agreement with experiment. This could be due to the indirect comparison made with the experimental data based on the calculated quadrupole deformation. However, the mac-mic potential models, which are treated in the same way, give an overall much better agreement. We note that Kortelainen et al., in their study of global nuclear properties and shell structure [36], conclude that the standard Skyrme energy density has reached its limits, and significant changes to the form of the functional are needed. 


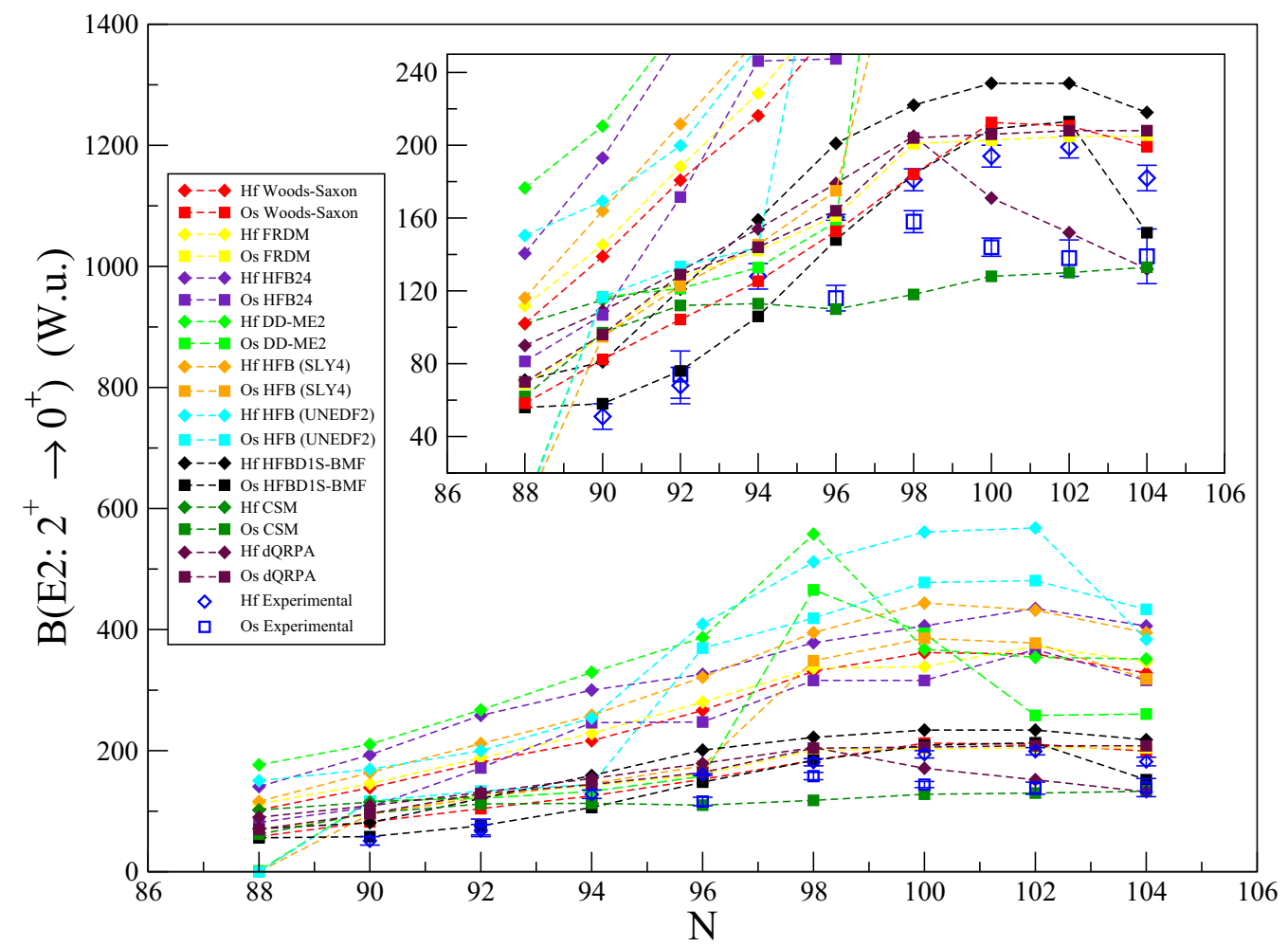

FIG. 3. Same as Fig. 2 (bottom) for the Hf and Os isotopic chains for neutron numbers from $N=88$ to $N=104$. The experimental $B(E 2)$ values for the Hf isotopes have been taken from Ref. [5] and references therein while the data for ${ }^{168} \mathrm{Os},{ }^{172} \mathrm{Os},{ }^{174} \mathrm{Os},{ }^{176} \mathrm{Os}$, and ${ }^{178,180} \mathrm{Os}$ come from Refs. [46-50], respectively.

\section{SUMMARY}

The lifetimes of the first excited $2^{+}$states in the extremely neutron-deficient nuclei ${ }^{162} \mathrm{~W}$ and ${ }^{164} \mathrm{~W}$ have been measured using the recoil distance Doppler shift technique. The evolution of collectivity in the neutron-deficient $\mathrm{W}$ isotopes from ${ }^{162} \mathrm{~W}$ and ${ }^{164} \mathrm{~W}$ with $N=88$ and $N=90$ to the neutron midshell at $N=104$, in terms of the reduced $E 2$ transition strength, has been investigated. We have compared the experimental data with the predictions of ground-state nuclear deformations and E2 transition strengths (when available) from various state-of-the-art theoretical models. In general, large deviations from the experimental data and even larger variations between the model predictions are found, accentuating the importance of electromagnetic transition strengths as a crucial probe for decisive tests of nuclear theory. Among the theoretical models investigated the Hartree-Fock-Bogoliubov beyond-mean-field calculation using the generator coordinate method based on the Gogny D1S interaction [43] stands out. It provides good predictions for $2_{1}{ }^{+}$excitation energies and the overall best predictions for the $B\left(E 2: 2^{+} \rightarrow 0^{+}\right)$reduced transition strength. However, none of the models tested was able to reproduce the experimental variation of $B(E 2)$ strength as a function of neutron number. An extension of the study to the Hf and Os isotopic chains revealed similar results, indicating that currently available models fail to quantitatively and qualitatively describe the collective behavior of these deformed rare-earth isotopes.

\section{ACKNOWLEDGMENTS}

This work was supported by the Swedish Research Council under Grant No. 621-2014-5558, the United Kingdom Science and Technology Facilities Council (STFC), the EU 7th Framework Programme, Integrating Activities Transnational Access, Project No. 262010 ENSAR, and the Academy of Finland under the Finnish Centre of Excellence Programme (Nuclear and Accelerator Based Physics Programme at JYFL). The authors acknowledge the support of the GAMMAPOOL for the JUROGAM detectors.
[1] S. Raman et al., At. Data Nucl. Data Tables 36, 1 (1987).

[2] S. Raman et al., At. Data Nucl. Data Tables 78, 1 (2001).

[3] L. Grodzins, Phys. Lett. B 2, 88 (1962).

[4] J. Zhang, R. F. Casten, A. Wolf, Z. Berant, R. B. Cakirli, N. V. Zamfir, and E. A. McCutchan, Phys. Rev. C 73, 037301 (2006).
[5] V. Werner, N. Cooper, J.-M. Régis, M. Rudigier, E. Williams, J. Jolie, R. B. Cakirli, R. F. Casten, T. Ahn, V. Anagnostatou, Z. Berant, M. Bonett-Matiz, M. Elvers, A. Heinz, G. Ilie, D. Radeck, D. Savran, and M. K. Smith, Phys. Rev. C 93, 034323 (2016).

[6] F. Beck et al., Prog. Part. Nucl. Phys. 28, 443 (1992). 
[7] C. Beausang and J. Simpson, J. Phys. G 22, 527 (1996).

[8] M. Leino et al., Nucl. Instrum. Methods Phys. Res., Sect. B 99, 653 (1995).

[9] M. J. Taylor et al., Nucl. Instrum. Methods Phys. Res., Sect. A 707, 143 (2013).

[10] R. D. Page et al., Nucl. Instrum. Methods Phys. Res., Sect. B 204, 634 (2003).

[11] J. Simpson, Z. Phys. A 358, 139 (2014).

[12] T. K. Alexander and J. S. Foster, Adv. Nucl. Phys. 10, 197 (1978).

[13] P. Petkov et al., Nucl. Instrum. Methods Phys. Res., Sect. A 431, 208 (1999).

[14] P. Petkov et al., Nucl. Instrum. Methods Phys. Res., Sect. A 560, 564 (2006).

[15] A. Dewald, S. Harissopulos, and P. von Brentano, Z. Phys. A 334, 163 (1989).

[16] P. Rahkila et al., Nucl. Instrum. Methods Phys. Res., Sect. A 595, 637 (2008).

[17] F. Seiffert, Program APATHIE, Institut für Kernphysik, Universitat zu Köln, 1989 (unpublished).

[18] D. C. Radford et al., Nucl. Instrum. Methods Phys. Res., Sect. A 361, 297 (1995).

[19] T. Kibedi et al., Nucl. Instrum. Methods Phys. Res., Sect. B 589, 202 (2008).

[20] H. J. Li et al., Phys. Rev. C 92, 014326 (2015).

[21] R. G. Helmer, Nucl. Data Sheets 101, 325 (2004).

[22] K. H. Schmidt et al., Phys. Lett. B 168, 39 (1986).

[23] R. S. Simon et al., Z. Phys. A 325, 197 (1986).

[24] E. S. Paul, P. J. Woods, T. Davinson, R. D. Page, P. J. Sellin, C. W. Beausang, R. M. Clark, R. A. Cunningham, S. A. Forbes, D. B. Fossan, A. Gizon, J. Gizon, K. Hauschild, I. M. Hibbert, A. N. James, D. R. LaFosse, I. Lazarus, H. Schnare, J. Simpson, R. Wadsworth, and M. P. Waring, Phys. Rev. C 51, 78 (1995).

[25] D. T. Joss et al., Phys. Rev. C 93, 024307 (2016).

[26] T. Back et al., Phys. Rev. C 84, 041306(R) (2011).

[27] A. Bohr and B. R. Mottelson, Nuclear Structure (World Scientific, Singapore, 1998), Vol. II, 45.

[28] J. Dudek, Z. Szymański, T. Werner, A. Faessler, and C. Lima, Phys. Rev. C 26, 1712 (1982).
[29] R. Wyss (private communication).

[30] Z. X. Xu and C. Qi, Phys. Lett. B 724, 247 (2013).

[31] Z. Y. Wu, C. Qi, R. Wyss, and H. L. Liu, Phys. Rev. C 92, 024306 (2015).

[32] P. Möller et al., At. Data Nucl. Data Tables 109-110, 1 (2016).

[33] E. Chabanat et al., Nucl. Phys. A 635, 231 (1998).

[34] M. Kortelainen, T. Lesinski, J. More, W. Nazarewicz, J. Sarich, N. Schunck, M. V. Stoitsov, and S. Wild, Phys. Rev. C 82, 024313 (2010).

[35] M. Kortelainen, J. McDonnell, W. Nazarewicz, P.-G. Reinhard, J. Sarich, N. Schunck, M. V. Stoitsov, and S. M. Wild, Phys. Rev. C 85, 024304 (2012).

[36] M. Kortelainen, J. McDonnell, W. Nazarewicz, E. Olsen, P.-G. Reinhard, J. Sarich, N. Schunck, S. M. Wild, D. Davesne, J. Erler, and A. Pastore, Phys. Rev. C 89, 054314 (2014).

[37] M. V. Stoitsov et al., Comput. Phys. Commun. 184, 1592 (2013).

[38] T. Nikšić et al., Comput. Phys. Commun. 185, 1808 (2014).

[39] G. A. Lalazissis, T. Niksic, D. Vretenar, and P. Ring, Phys. Rev. C 71, 024312 (2005).

[40] S. Goriely et al., Phys. Rev. C 88, 024308 (2013).

[41] D. S. Delion and J. Suhonen, Phys. Rev. C 87, 024309 (2013)

[42] D. S. Delion and A. Dumitrescu, At. Data Nucl. Data Tables 101, 1 (2015).

[43] G. F. Bertsch, M. Girod, S. Hilaire, J. P. Delaroche, H. Goutte, and S. Peru, Phys. Rev. Lett. 99, 032502 (2007).

[44] D. S. Delion (private communication).

[45] B. Sayği, Ph.D. thesis, University of Liverpool, 2014 (unpublished).

[46] T. Grahn et al., Phys. Rev. C 94, 044327 (2016).

[47] A. Virtanen et al., Nucl. Phys. A 591, 145 (1995).

[48] C. B. Li, X. G. Wu, X. F. Li, C. Y. He, Y. Zheng, G. S. Li, S. H. Yao, S. P. Hu, H. W. Li, J. L. Wang, J. J. Liu, C. Xu, J. J. Sun, and W. W. Qu, Phys. Rev. C 86, 057303 (2012).

[49] B. Melon, Ph.D. thesis, University of Cologne, 2011 (unpublished).

[50] O. Möller, P. Petkov, B. Melon, A. Dewald, A. Fitzler, J. Jolie, D. Tonev, S. Christen, B. Saha, K. O. Zell, and M. Heidemann, Phys. Rev. C 72, 034306 (2005). 